\title{
Metabolomics reveals altered lipid metabolism in a mouse model of endometriosis
}

\author{
Mainak Dutta ${ }^{1,3}$, Mallappa Anitha ${ }^{1}$, Philip B. Smith ${ }^{2}$, Christopher R. Chiaro ${ }^{1}$, \\ Meenu Maan ${ }^{4}$, Koel Chaudhury ${ }^{3}$, Andrew D. Patterson ${ }^{1,2, *}$ \\ ${ }^{1}$ Center for Molecular Toxicology and Carcinogenesis, Department of Veterinary \& Biomedical \\ Sciences, Pennsylvania State University, University Park, PA 16802, USA \\ ${ }^{2}$ The Huck Institutes of the Life Sciences, Pennsylvania State University, University park, PA, \\ 16802, USA \\ ${ }^{3}$ School of Medical Science and Technology, Indian Institute of Technology Kharagpur, \\ Kharagpur, WB 721302, India \\ ${ }^{4}$ School of Biotechnology, Jawaharlal Nehru University, New Delhi, Delhi 110067, India
}

Table of Contents for Supporting Information

Table S1: List of serum lipids identified in positive mode Table S2: List of serum lipids identified in negative mode Table S3: List of liver lipids identified in positive mode Table S4: List of liver lipids identified in negative mode Table S5: Total liver PC/PE calculation 